\title{
Calendario civile: gli studenti raccontano gli anni Settanta. Un progetto di alternanza scuola-lavoro
}

Riflessioni su un progetto di alternanza scuola-lavoro, organizzato dall'Isrec Parma, e sulle problematiche relative all'insegnamento della storia più recente nelle scuole.

Presentation of an educational project, organized by Isrec Parma, and reflections on the problems related to the teaching of the most recent Italian history in schools.

\section{II progetto Calendario civile}

L'Istituto della Resistenza e dell'età contemporanea (Isrec) di Parma ha tentato di affrontare quest'anno, attraverso un progetto di alternanza scuola-lavoro rivolto a studenti delle ultime due classi di due istituti superiori della nostra città, un nodo problematico della didattica della storia nelle scuole: l'insegnamento della più recente storia repubblicana. Con il coordinamento dei ricercatori dell'istituto Carlo Ugolotti e Domenico Vitale, si è quindi pensato di organizzare un percorso formativo didattico seguito da una parte "laboratoriale-realizzativa", che desse ai partecipanti stessi un ruolo attivo nella realizzazione di una restituzione scenica. Tenendo conto del fatto che tendenzialmente l'insegnamento liceale della storia - per una varietà di cause che vanno dallo scarso tempo disponibile ad altri impedimenti di ordine organizzativo - si ferma alla Seconda guerra mondiale, si è tentato di costruire un progetto formativo intorno alla nozione di "calendario civile" [Portelli 2017]. In occasione del "Giorno della memoria dedicato alle vit- 
time del terrorismo", si è pensato di incentrare il percorso sulla storia degli anni Settanta, stagione particolarmente problematica dal punto di vista della didattica per una serie di ragioni: una mancanza di approfondimento storiografico degli inseganti rispetto al periodo preso in esame; il peso di giudizi personali, talvolta legati al proprio vissuto personale; il prevalere di un discorso giornalistico nel dibattito pubblico.

La mancanza di "alfabetizzazione storica" degli studenti è stato perciò il primo problema da affrontare in sede progettuale: attraverso le passate esperienze di collaborazione tra l'istituto e le scuole, si è infatti potuto notare come tendenzialmente i partecipanti ai progetti avessero più familiarità con altri periodi storici, quali la Seconda guerra mondiale o il Risorgimento - eventi ben più presenti nel calendario delle celebrazioni e ricorrenze istituzionali - , rispetto al passato a loro più prossimo. A tal proposito prima di affrontare due casi specifici si è pertanto scelto di fare introdurre a due giovani storici, Alfredo Mignini (dottore di ricerca presso l'Università di Bologna) e Alessia Masini (dottoressa di ricerca presso l'Università di Macerata), una contestualizzazione panoramica degli anni Settanta. Il primo ha presentato questo decennio da un punto di vista politicoistituzionale, la seconda da un punto di visto sociale e culturale. È così emersa la distanza degli studenti da termini come "partito di massa", "centrosinistra" e

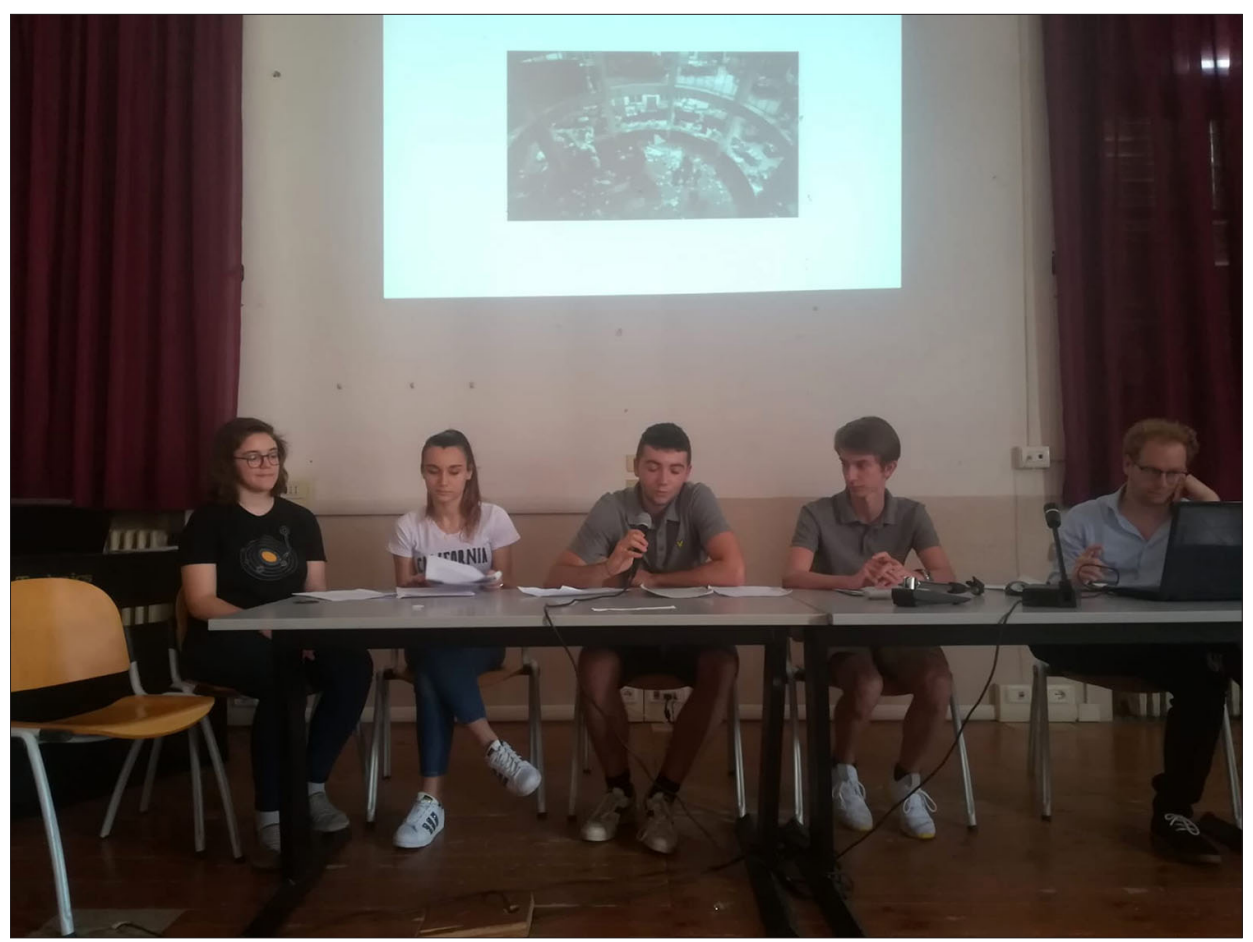

Gruppo di studenti al lavoro sulla strage di Bologna 
finanche "guerra fredda". Affrontando invece gli aspetti culturali e di produzione mass-mediale si è notato come musica, cinema e moda siano molto più familiari nell'immaginario contemporaneo degli studenti. Dopo gli interventi iniziali, si è scelto di dividere i partecipanti in due gruppi distinti per lavorare su due casi specifici in modo che, nel corso della fase finale di restituzione, il quadro sugli anni Settanta fosse il più completo possibile, pur partendo da esempi concreti $\mathrm{e}$ particolari: la strage di Bologna e l'omicidio di Walter Tobagi.

Per quanto riguarda il primo gruppo la parte didattica è stata così organizzata: una lezione di Cinzia Venturoli per presentare il problema e le tematiche legate allo stragismo e all'eversione nera; una visita a Bologna sui luoghi della strage; un incontro con un testimone (in ambedue i casi guidati da Venturoli) e una visita alla sezione audiovisiva dell'Istituto Parri per visionare il film di Giampaolo Bernagozzi Dedicato a chi perdona, introdotto da Luisa Cigognetti. La stessa Venturoli ha poi presentato agli studenti i fatti della strage e le dinamiche processuali che ne sono seguite. Infine, ha chiuso la parte formativa un laboratorio, tenuto da Carlo Ugolotti, sulla rappresentazione della strage al cinema utilizzando il film come fonte storica, vettore di memoria (a volte mistificante, si veda Romanzo criminale di Michele Placido, 2005) e strumento storiografico.

Il secondo gruppo invece ha partecipato a una lezione di Cinzia Venturoli sul terrorismo rosso; a un'analisi di Toni Rovatti delle vicende processuali del caso Tobagi; a un laboratorio tenuto da Carlo Ugolotti sulla rappresentazione delle

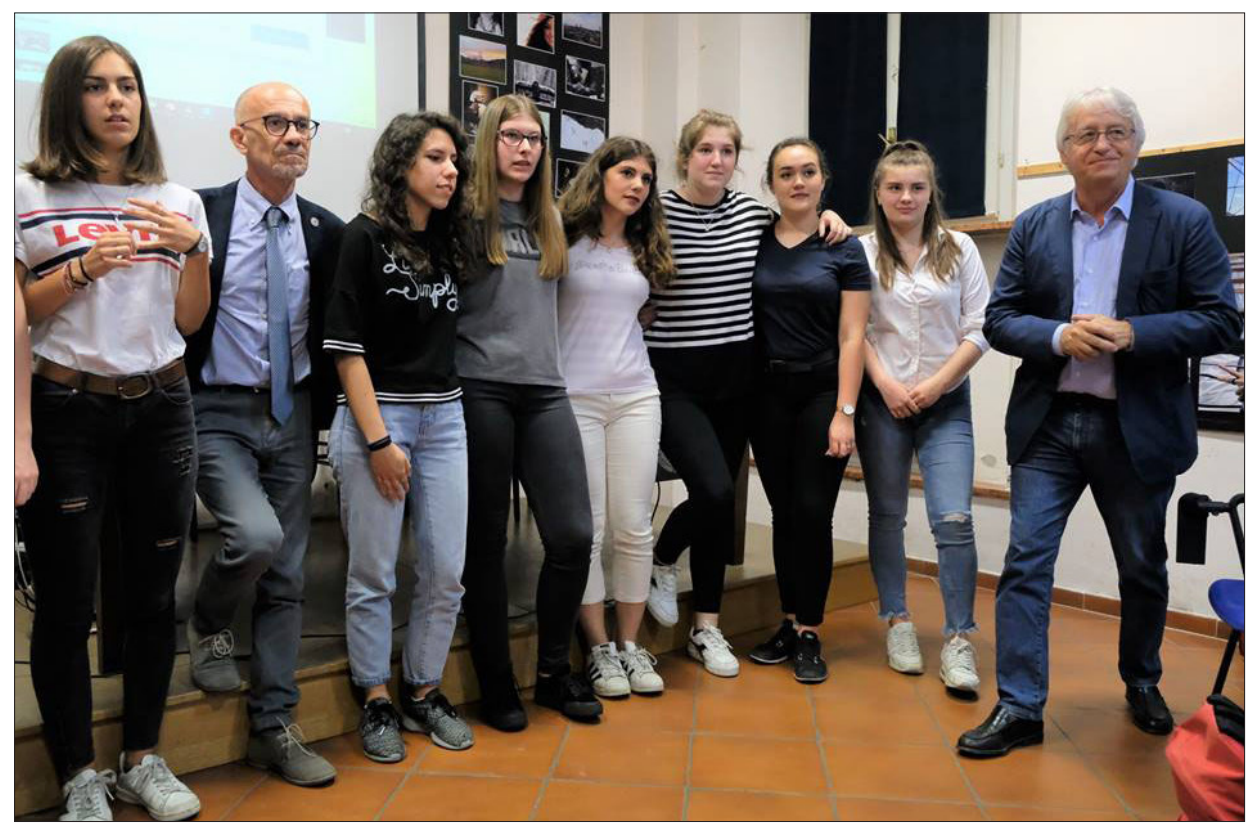

Gruppo di studenti al lavoro sul caso Tobagi 
Brigate Rosse e, più in generale, sul clima degli anni Settanta al cinema. Il percorso si è concluso con un incontro con Benedetta Tobagi, figlia di Walter ma anche storica e autrice del volume Come mi batte forte il cuore: storia di mio padre [Tobagi 2009].

Nonostante qualche ritrosia iniziale degli studenti e le evidenti difficoltà di mediazione tra un linguaggio di tipo accademico e le esigenze di coinvolgimento degli studenti, i partecipanti si sono via via appassionati ai due casi studio, soprattutto dopo aver familiarizzato con gli ambienti storico-culturali di riferimento: determinanti in questo senso sono stati l'incontro con i testimoni e la visita a Bologna, che hanno dato alla narrazione storica una dimensione di emozionalità senza tuttavia mai rinunciare alla "scientificità" del discorso, attribuendo sostanza concreta e viva a luoghi e protagonisti della Storia.

A seguito della fase formativa ai partecipanti è stato chiesto di realizzare due incontri pubblici aperti alla cittadinanza, in cui raccontassero i casi di studio da loro esaminati, non attraverso una mera esposizione dei fatti ma attraverso una rielaborazione personale di quanto appreso. La gestione degli incontri laboratoriali ha presentato alcune difficoltà in quanto gli studenti non avevano troppa familiarità con la costruzione di una "conferenza scenica" e una certa ritrosia a fornire pubblicamente interpretazioni personali. Inoltre, un fattore di ulteriore inibizione degli studenti poteva dipendere dal fatto di trovarsi in gruppi composti da ragazzi provenienti da altre classi o scuole. Tuttavia, una volta indirizzati e sciolte le prime ritrosie, i partecipanti hanno lavorato con grande impegno per dare forma alle presentazioni finali.

\section{Le restituzioni}

La restituzione finale del gruppo che ha analizzato il caso dell'omicidio di Tobagi -presentato nell'aula magna del Liceo Scientifico "G. Marconi" il 30 maggio 2018 - è stata introdotta dagli interventi del prefetto di Parma Giuseppe Forlani, del dirigente scolastico Adriano Cappellini, del presidente dell'Isrec Attilio Ubaldi e del direttore Marco Minardi.

La conferenza scenica era articolata in unità autonome che, pur nella loro frammentarietà, offrivano un efficace quadro d'insieme sulla violenza politica e sulla cultura degli anni Settanta. Raccordate da canzoni dell'epoca, gli studenti hanno illustrato il contesto storico e sociale del decennio (Jora Ndreu e Virginia Monica), ricostruito la vicenda e gli atti processuali (Valeria Dogotec e Chiara Maccaferri), simulato un dialogo immaginario tra un giovane di allora e uno di oggi (Andrea Chiesa, Francesca Garritano e Alice Ferrari) e presentato considerazioni 
finali sul tema della giustizia e del perdono, terminando con una serie di domande aperte rivolte al pubblico (Anna Butnari e Catalina Melnic).

La restituzione finale del gruppo che si è occupato della strage di Bologna - presentato nell'aula magna del Liceo Classico “G. D. Romagnosi” il 31 maggio 2018 - era articolata più o meno come quella che l'aveva preceduta. Virginia Stevenin (III A del Romagnosi), Alexa Mastaj (II G del Romagnosi) e Elena Agnesini hanno raccontato il contesto storico partendo dal 1968 attraverso l'analisi di fotografie d'epoca. Jacopo Artoni e Giacomo Abbati Brunazzi (entrambi della II A del Romagnosi) hanno illustrato al pubblico la storia e gli obbiettivi dei gruppi eversivi di destra. Giada Vincetti (II D del Romagnosi), Xhoana Kaja (II G del Romagnosi) ed Emma Romanini (IV M del Marconi) hanno narrato, come fosse un reportage giornalistico, le vicende processuali. Lara dell'Acqua, Asia Pezziga (IV M del Marconi) e Giada Ricci (IV K del Marconi) hanno riflettuto sulla trasmissione della memoria (o la mancanza della stessa) attraverso i canali istituzionali e i media popolari, realizzando anche un'inchiesta tra i cittadini di Parma e i loro coetanei verificando l'effettiva conoscenza riguardo la strage. Miriam Piantoni e Sara Bernardelli hanno raccontato le biografie di alcune vittime e presentato delle riflessioni finali sul senso della violenza e sulle ricadute di quest'ultima nella vita e nelle famiglie degli innocenti coinvolti.

In entrambi i casi i temi che hanno più coinvolto emotivamente gli studenti sono stati quelli della giustizia, del perdono e del rapporto tra familiari delle vittime e sopravvissuti, con i "responsabili" analizzati anche grazie al ricorso e al raffronto delle conoscenze letterarie acquisite durante l'anno scolastico (usando tra gli altri, come termine di paragone la tragedia greca). Ancora una volta si può quindi intravedere in sottotraccia la relativa distanza delle giovani generazioni attuali dalle tematiche e dai linguaggi dell'attivismo politico degli anni Settanta: i ragazzi percepiscono più vicine a loro tematiche morali universali, rispetto a quelle legate alla concreta specificità storica del decennio preso in considerazione.

Al termine di entrambe le presentazioni, alcuni docenti delle scuole coinvolte hanno riflettuto su come e cosa significhi insegnare oggi la storia dell'Italia repubblicana.

\section{Considerazioni finali}

Si è quindi cercato nel corso di questo intero progetto di presentare un ritratto completo di quel decennio, aldilà di facili formule ("anni di piombo"), banalizzazioni, giudizi politici, cercando di colmare un vuoto la cui responsabilità ricade sugli insegnanti ma anche sulle istituzioni e sugli stessi storici che non hanno 
saputo costruire un ponte tra gli studenti e il nostro passato più vicino sia da un punto di vista cronologico che geografico.

Gli anni Settanta sono infatti stati marcati non solo da un'esplosione della violenza nell'attivismo politico, ma anche da sperimentalismi culturali e da grandi conquiste nel campo dei diritti civili e sociali, nonché da una presa di coscienza verso una serie di soggetti in precedenza messi in secondo piano. A tal proposito, una riflessione fatta dagli studenti durante la restituzione pubblica ha evidenziato che sarebbe ingiusto parlare di questo decennio limitandosi alla violenza. Sono stati anni segnati anche da importanti riforme nella società italiana, come l'approvazione del nuovo diritto di famiglia, delle leggi sul divorzio e sull'aborto, della legge Basaglia che portò alla chiusura dei manicomi. Inoltre sono stati anni in cui il fermento culturale si esprimeva nella musica internazionale ma non di meno in quella italiana: si pensi per esempio a Fabrizio De André e agli squarci sociali raccontati nelle sue canzoni.

Per avvicinare gli studenti alla storia più prossima a noi - ma tuttavia relegata ai margini dall'insegnamento tradizionale - è forse necessario inquadrare gli anni Settanta non solo come periodo di violenza e stragi, ma inserendo questi fenomeni all'interno di una cornice più ampia che includa la dimensione culturale e sociale, permettendo alle nuove generazioni di comprendere come in quel decennio vennero enucleati e originati tanti caratteri e peculiarità della nostra società attuale: nuovi diritti, legislazioni, rapporti (anche di sfiducia) dei cittadini nei confronti delle istituzioni, forme di attivismo politico, gusti e mode del consumo. Si tratta dunque di assumere una prospettiva di longue durée per leggere il presente, in modo da permettere agli alunni di vedere come tanti dei problemi attuali abbiano le loro radici nel nostro passato più o meno vicino. La riduttiva formula degli "anni di piombo", oltre che storiograficamente banalizzante, risulta una gabbia che rischia di allontanare l'interesse delle nuove generazioni da un importante decennio della storia repubblicana.

\section{Bibliografia essenziale per la parte formativa}

Arlati R. e Magosso R. 2003, Le carte di Moro, perché Tobagi: chi portò via gli scritti caldi di Aldo Moro: $i$ nomi, i reati, i retroscena: come e quando decisero di non salvare Walter Tobagi, Milano: F. Angeli

Brambilla M. 1993, L'Eskimo in redazione. Quando le Brigate rosse erano "sedicenti", Milano: Bompiani

Flores M. e Gallerano N. 1992, Sul PCI: un 'interpretazione storica, Bologna: Il Mulino 
Galfré M. 2014, La guerra è finita. L'Italia e l'uscita dal terrorismo, 1980-1987, RomaBari: Laterza

Lanaro S. 1996, Storia dell'Italia repubblicana, Venezia: Marsilio

Peretti L. e Roghi V. 2014, Immagini di piombo. Cinema, storia e terrorismi in Europa, postmedia books

Portelli A. 2017, Calendario civile. Per una memoria laica, popolare e democratica degli italiani, Donzelli: Roma

Scorti P. V. 2003, L'affaire Tobagi: un giallo politico, Milano: Montedit

Tobagi B. 2009, Come mi batte forte il cuore: storia di mio padre, Torino: Einaudi

Uva C. 2007, Schermi di piombo: il terrorismo nel cinema italiano, Soveria Mannelli: Rubbettino

Venturoli C. 2012, Stragi fra memoria e storia. Piazza Fontana, Piazza della Loggia, la stazione di Bologna: dal discorso pubblico all'elaborazione didattica, Sette città: Viterbo 